\title{
Effect Inclusion of Graphene on the Mechanical Properties of Polymer Nanocomposites
}

\author{
Nur Aqilah Sairy, Norkhairunnisa Mazlan, Mohamad Ridzwan Ishak , Nik Noriman Zulkepli
}

\begin{abstract}
Graphene-based nanocomposites have introduced to the development of flexible nanocomposite as for emerging applications like as in need of superior of thermal, mechanical, electrical, chemical and optical performance. Sp2-hybridized carbon atoms are arranged in a two-dimensional lattice which is a monolayer that produces the graphene. Graphene has gained significantly as fillers in the nanocomposite due to it has various intriguing properties. This review will discuss the effect of inclusion of graphene on the mechanical properties of polymer nanocomposites.
\end{abstract}

Index Terms: mechanical properties; nanocomposites; nanofiller; graphene

\section{INTRODUCTION}

Class of materials such as nanocomposite materials with nanoscale fillers has emerged in the past decades. It has great advantages in increased specific interfacial area, controlled interfacial interactions, higher achievable loads, and higher overall compliance. The interfacial area between the filler and the matrix is maximized as the mismatch of the physical properties of the components becomes much less critical. As a result, the interfacial strength can be enhanced [1]. Presently, there are nanofillers that can give a huge enhancement to the mechanical performance of multifunctional nanocomposites [2][4], which were the inorganic nanoparticles, metal nanostructures, and the pre-treated carbon nanotubes [2][3]. Graphene has better dispersion in a polymer matrix and also has large aspect ratios that act as an advantage to enhance properties [5][6]. Carbon-based nanofillers such as carbon nanotubes (CNTs) and graphene derivatives have been reported that it can improve the gas barrier, thermal and mechanical properties of the polymers due to the high strength on the amount of sp2 bonded carbon atoms [6][7].

Revised Manuscript Received on July 05, 2019.

Nur Aqilah Sairy, Department of Aerospace Engineering, Faculty of Engineering, UPM, 43400 UPM Serdang, Selangor Darul Ehsan, Malaysia.

Norkhairunnisa Mazlan, Department of Aerospace Engineering, Faculty of Engineering, UPM, 43400 UPM Serdang, Selangor Darul Ehsan, Malaysia, \& Higher Institution Center of Excellence (HiCoE), Institute of Tropical Forestry and Forest Products (INTROP), Universiti Putra Malaysia, 43400 UPM, Serdang, Selangor. Corresponding author. Email: norkhairunnisa@upm.edu.my

Mohamad Ridzwan Ishak, Department of Aerospace Engineering, Faculty of Engineering, UPM, 43400 UPM Serdang, Selangor Darul Ehsan, Malaysia.

Nik Noriman Zulkepli, a Center of Excellence Geopolymer and Green Technology (CeGeoGTech), Faculty of Engineering Technology, Universiti Malaysia Perlis, 02100 Padang Besar, Perlis, Malaysia.
About 2 to $5 \mathrm{wt} \%$ has been varied for the effective percolation threshold of CNTs in the polymer composites. Nevertheless, the percolation threshold of graphene and its derivatives lies in the range of 0.5 to $2 \mathrm{wt} \%$.

Recently, researchers and industrialists have been attracted and started to give attention to the graphene as it can create an extreme achievement in the properties at very low fillers loading [8][9].

\section{GRAPHENE NANOFILLER}

Graphene has one atom thick planar sheet of $\mathrm{sp} 2$ bonded carbon atoms and it is a two-dimensional carbon nanofillers that is tightly packed in a crystal lattice of honeycomb. It is regarded the "thinest material in the world" with huge application potential for implementation [10][11]. Testing properties of graphene-reinforced polymer nanocomposites for instance the electrical, thermal, and mechanical are rely on the differences of weight fraction, topographical features, dispersion status, surface functionality and morphology of graphene [12][13].

When a polymer is strengthened with the graphene, the carbonaceous sheets will shackle the cracks and limit their progress.. Graphene at the interface making this deflection and obstruction is mainly a mechanism to increase nanocomposite fracture toughness [14]. An improvement in the mechanical and thermal characteristics of multiple differences polymer nanocomposites for instance Polyethylene Terephthalate (PET), Polyvinyl alcohol (PVA), and Poly-methyl-methacrylate (PMMA) and epoxy nanocomposites has been reported following the incorporation of graphene derivatives [15][16][17][18].

The nanocomposite manufacturing handling circumstances are the major factor that can influence the ultimate characteristics of graphene-based polymer nanocomposites [5][19]. The primary role of graphene parts is to lower the loading content, making it more extremely distributed and structured sheets within the polymer matrix to improve nanocomposite's general characteristics. Component affinity, interfacial strength, dispersion, and spatial organization all play a major role in determining the toughness, rigidity, elongation, strength of polymer nanocomposites under different loading circumstances. In particular, the mechanical characteristics for instance ultimate tensile strength and strain, flexural strength, and Young's modulus depends on the specific surface area, organisation, loading rate and




aspect ratio of graphene materials. [20][21].

\section{MECHANICAL PROPERTIES OF GRAPHENE}

Inclusion of graphene in the form of sheets provides enhancement to the mechanical characteristics in polymer-based nanocomposites [22][23]. To give an instance, the mechanical characteristics of resultant nanocomposites with various graphene contents were examined once oxygenated graphene sheet partially dispersed in the polymer matrix [24]. The analysis found that the Young modulus, mode I fracture toughness (KIC) and ultimate tensile strength of the nanocomposite with $0.125 \%$ weight fractions are $50 \%$, $65 \%$, and $45 \%$ larger than those of its graphene free counterpart (the baseline epoxy), respectively. The ideal nanofiller component for high performance and multi-functional polymer nanocomposites with high toughness fabrication is made from graphene-based derivative that are mechanically strong but flexible [5][10].

The molecular interactions with polymers are limited to weak van der Waals forces, hydrophobic-hydrophobic interactions, and $\pi-\pi$ stacking due to the homogeneous carbon structure of graphene without additional heteroatom [25]. Van der Waals is universal attractive interactions between the molecules generated by permanent or transient dipoles of the molecules. In spite of the fact that it is very weak, these forces giving major part interfacial strength between the common polymer and graphene for instance polyethylene which is due to it has the very large specific surface area and intimate contact [26]. From [10] been stated that graphene-based polymer nanocomposites show superior mechanical properties compared to the neat polymer or conventional graphite based composites. Interfacial interactions between polymers and graphene-based materials are an important factor that plays a key role in the mechanical integrity of the corresponding nanocomposite and their mechanical performance [27]. For binding graphene in the hydrophobic polymer matrices are there's must have the interaction between the hydrophobic-hydrophobic. The $\pi-\pi$ interactions played an important factor for graphene materials with the electro rich aromatic rings interacting with a multitude of chemical species and matrices with phenyl rings, which can function as powrful bonding locations [28][29]. In addition, $\pi-\pi$ stacks can adapt to different kind of space organizations and significantly enhance the bonding in graphene nanocomposites.

High-performance

graphene-based

carbon nanofiller/polymer composites for piezoresistive sensor applications was been experimenting [30]. Figure 1 shows the mechanical properties of the composites that were analyzed by uniaxial stress-strain measurements until the sample ruptured. As shown in figure 1A, it illustrates the typical behavior of the materials which are the properties of the reduced graphene oxide (rGO) / Poly (vinylidene fluoride) (PVDF) composites as a function of the different nanofillers content that varies from $0.25 \mathrm{wt} \%$ until $5 \mathrm{wt} \%$. Figure $1 \mathrm{~B}$ presenting the stress-strain data which is acquired from the composites with $5 \mathrm{wt} \%$ of the different nanofillers.

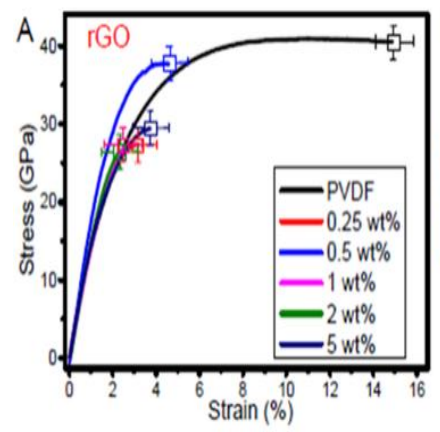

(a)



(b)
Fig. 1: Mechanical curve measurements of stress-strain at $1 \mathrm{~mm} / \mathrm{min}$ as a function of the nanofillers type and loading content for A) rGO composites and B) $5 \mathrm{wt} \%$ of carbonaceous nanofillers with composites (SWCNH, GO, G-NPL and rGO).

The maximum stress for pristine PVDF sample as illustrated from the graph above is about $40 \mathrm{GPa}$. It is because composites generally present lower values, decreasing with the increase of nanofiller content, reaching a minimum of about $25 \mathrm{GPa}$ for the sample with $5 \mathrm{wt} \%$ single-walled carbon nanohorns (SWCNH).

Figure 1A shows the stress-strain curves which are the typical mechanical behavior of a thermoplastic. It has been reported in [31] that the maximum strain of the composites will be decreased when there incorporation of the carbonaceous nanofillers into the PVDF matrices. It is characterized by the elastic region with linear behavior in which Young's modulus is calculated (slope of the stress-strain curve until\% of deformation) and the plastic region after the yielding zone [31].

The Young's modulus of pristine PVDF is $1.16 \pm 0.03 \mathrm{GPa}$ and $I$ the values obtained for the composites are similar among different samples ranging between (1.10-1.70) \pm 0.03 $\mathrm{GPa}$. Furthermore, it is also presenting an increment trend with nanofiller content which is increased up to $5 \mathrm{wt} \%$. The maximum strain for the various composites is between 2 to $5 \%$ while 16 to $18 \%$ for the pristine PVDF sample.

The experiment effect of Graphene Nanofiller on mechanical properties of Acrylonitrile Butadiene Styrene (ABS)/Graphene Nanocomposites was been prepared [32]. Mechanical properties of compressive specimens of Graphene/ABS nanocomposites was studied in this present study. Young modulus from the strength and strain graphs for the compression which is at the different of loading content is studied and the results are illustrated as shown in Figure 2. The author finds out that the graph shows the Young modulus is increasing to $37.83,26.21$, and $18.43 \%$ respectively as the percentage of graphene content is increased (from $2.5 \%, 5 \%$ to $7.5 \%$ ). The values reveal that the mechanical properties of ABS nanocomposites are significantly enhanced as the graphene content is increasing. This is because of the value of Young modulus of compression is increasing. The different samples of compressive strength are studied under the strain rate which is $2.5 s^{-1}$. The dimension of the compressive sample is about $5 \mathrm{~mm}$ in width and $10 \mathrm{~mm}$ in diameter. 


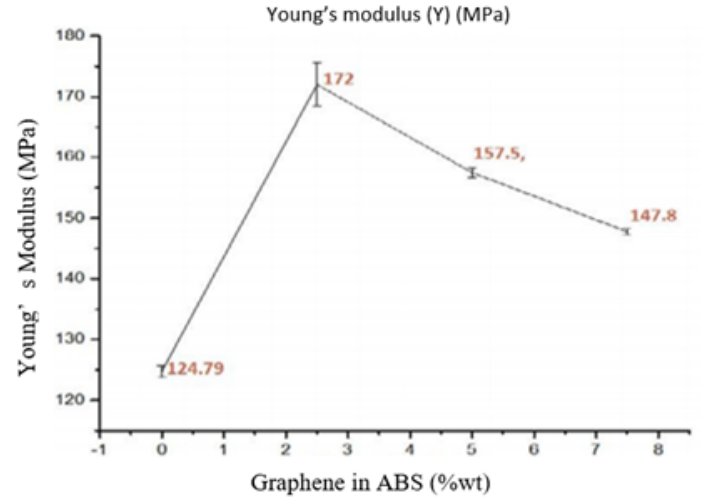

Figure 2: Different loading content of graphene for Young's Modulus (MPa) graph.

As per the data, the graph is plotted and it is indicated that the lower percentage is more significantly good compared to the higher percentage as it can enhance Young's modulus value. The value of pure ABS in Young's Modulus's reading is $124.79 \mathrm{MPa}$. Its value is increased to $172,157.5$ and 147.8 (MPa) at 2.5\%, 5\% and 7.5 respectively as shown in table 2 . The Young's modulus and its percentage increment in comparison to pure ABS are increased to $37.83 \%$ at $2.5 \%$ graphene content. There is an increment in Young's Modulus value after the graphene loading is increasing to beyond $2.5 \%$ but it's still lesser extent. This is because of the lower wt\% loading proper distribution of graphene nanofillers in the ABS matrix. The real fact is when there is agglomerated of graphene, it will lead to decrease the tensile strength values. But if the graphene is uniformly well dissolved in the polymer matrix, it will definitely get higher tensile strength values. Despite the graphene nanofillers used less filler loading, it actually leads to higher enhancement in Young's modulus of compression as it is uniformly and well dissolved in the polymer matrix [18].

\section{APPLICATION}

In current studies, they are focusing the most important functionalities that can enhance the thermal, electrical, optical and barrier properties. The conductivity, percolation threshold and mechanical properties of the nanocomposites were analyzed and tested for prospective applications including biosensors, supercapacitors, gad electrodes and transparent conducting electrode [34][35][36]. Up to this point, graphene components, for instance, are poly (3, 4-ethyldioxythiophene), nafion, polyaniline (PANI), polystyrene (PS) and epoxy polymer have been included in a variety of polymer matrices to fabricate the nanocomposites with new functionalities [18][33].

Graphene has its own fascinating properties that can enhance several different types of applications. It is an eventually transparent, thin, flexible conductor, and mechanically very strong. Graphene also can be used in applications, for instance, solar cells, light panels and touch screens where it can replace the rather expensive and fragile Indium Tin Oxide (ITO), this is because graphene is a transparent conductor. The mobility of graphene is good which makes the material very interesting for electronic high-frequency applications [37][38]. The effect of quantum Hall in graphene could potentially make to an even more accurate the resistance standard in metrology [42]. By using this either two like an electrical field or chemical doping, it can modify over a large range for the conductivity. The other potential applications are gas sensors and flexible electronics [40][41]. Besides that, $70 \mathrm{~cm}$ of graphene sheet also can be produced by using the near-industrial method. This is because it has been reported that it is possible for fabricating the large sheet of graphene sample [39].

Graphene-based known as a new type of composite materials. It could also become interesting for use in the satellites and aircraft with the great strength and low weight of the graphene-based properties [43].

\section{CONCLUSION}

This paper reviewed the effect of inclusion of graphene on the mechanical properties of polymer nanocomposite. Graphene-based nanofillers have been shown the enhancing properties when their presence in the advanced engineering application. This is because this family of materials has possibilities and the capabilities practically endless due to their enhancing properties. There are still several challenges that should be stated and have to tackle before the mass production of graphene nanocomposites can proceed to the industry. For instance, focus on producing high-quality graphene so that it will lead to the higher properties of the materials which is one of the major issues been stated. Dispersion of the filler should be homogenous so that there are no aggregates in the composites as to get the successful production of the advanced composites. If there are aggregates in the composites, as a result, there will be a failure point while the testing procedure is held. Besides, the filler should not exhibit the characteristics which are it can limit its abilities such as impurities, wrinkles, defects or small lateral size. Other than that, there must have a strong bonding connection between the filler and the matrix so that it can get good efficient stress transfer.

\section{ACKNOWLEDGMENT}

I would like to first say a thank you to Dr. Norkhairunnisa Binti Mazlan who has supervised me with her motivation, patience, and immense knowledge. Her guidance helped me in all the time of the research. I would also like to show gratitude to my co-supervisor, including Dr. Mohamad Ridzwan Bin Ishak from University of Putra Malaysia (UPM) and Prof. Dr. Nik Noriman Bin Zulkepli from University of Malaysia Perlis (UniMAP). I gratefully acknowledge the grant received towards my research study from the Higher Institution Centre of Excellence (HiCOE). I am also very grateful to all those at the composite and Introp laboratory, who were always so helpful and provided me with their assistance throughout my research work.

\section{REFERENCES}

[1] P.M. Ajayan, L.S. Schadler, and P.V. Braun, "Related Titles from Wiley $\mathrm{VCH}$ Molecular Imprinting from Fundamentals to Applications Multilayer Thin Films Sequential Assembly of Nanocomposite Materials High-Temperature Ceramic Matrix Composites Nanotechnology”, 2005.

[2] H. Ko, C. Jiang, H. Shulha, and V.V. Tsukruk, (2005). Carbon nanotube arrays encapsulated into freely suspended flexible films. Chemistry of Materials, 17(10), 2490-2493.

[3] Q. Cheng, B. Wang, C. Zhang, and Z. Liang. (2010). Functionalized carbon nanotube sheet/bismaleimide nanocomposites. Mechanical and 
electrical performance beyond carbon fiber composites, 6(6), 763-767.

[4] A. Mamedov, and N.A. Kotov. (2000). Free Standing Layer by Layer Assembled Films of Magnetite Nanoparticles. Langmuir, 16(13), 5530-5533.

[5] M. Moniruzzaman, and K.I. Winey. (2006). Polymer nanocomposites containing carbon nanotubes. Macromolecules, 39, 5194-5205.

[6] J.C. Huang, (2002). Carbon black filled conducting polymers and polymer blends. 21, 299-313.

[7] S. Stankovich, D.A. Dikin, G.H.B. Dommett, K.M. Kohlhaas, E.J. Zimney, E.A. Stach, R.D. Piner, S.T. Nguyen, and R.S. Ruoff. (2006). Graphene-based composite materials. Nature, 442(7100), 282-286.

[8] Y. Sun, Y. Luo, and D. Jia. (2008). Preparation and properties of natural rubber nanocomposites with solid state organic modified montmorillonite. Journal of Applied Polymer Science, 107, 2786-2792.

[9] V. Sharma, M. Goyal, and P. Jindal. (2017). Effect of Graphene Nanofiller on Mechanical Properties of Acrylonitrile Butadiene Styrene (ABS)/Graphene Nanocomposites. Polymer Composites, 5(6), 507-512.

[10] T. Kuillaa, S. Bhadra, D. Yao, N.H. Kim, S. Bose, and J.H. Lee. (2010). Recent advances in graphene based polymer composites. Progress Polymer Science (Oxford), 35(11), 1350-1375.

[11] A.K. Geim, and A.H. MacDonald. (2007). Graphene: exploring carbon flatland. Physic Today, 60, 35-41.

[12] Y. Si, and T. Samulski. (2008). Synthesis of water soluble graphene. Nano Letters, 8, 1679-1682.

[13] R. Atif, I. Shyha, and F. Inam. (2016). Mechanical, Thermal and Electrical Properties of Graphene Epoxy Nanocomposite. A Review, Polymers, 8(080281), 1-37.

[14] J. Ma, Q. Meng, I. Zaman, S. Zhu, A. Michelmore, N. Kawashima, C.H Wang, and H.C. Kuan. (2014). Development of polymer composite using modified, high structural integrity graphene platelets. Composites Science and Technology, 9, 82-90.

[15] M. Cano, U. Khan, T. Sainsbury, A.O. Neill, Z. Wang, I.T. McGovern, W.K. Maser, A.M. Benito, and J.N. Coleman. (2013). Improving the mechanical properties of graphene oxide based materials by covalent attachment of polymer chains. Carbon, 52, 363-371.

[16] H.B. Zhang, W.G Zheng, Q. Yan, Y. Yang, J.W. Wang, Z.H. Lu, G.Y. Ji, and Z.Z. Yu. (2010). Electrically conductive polyethylene terephthalate/graphene nanocomposite prepared by melt compounding. Polymer, 51, 1191-1196.

[17] R.K. Layek, D.P. Samanta, D.P. Chatterjee, and A.K. Nandi. (2010). Physical and Mechanical Properties of Poly (methyl methacrylate)-functionalized graphene / Poly (vinylidine Fluoride) nanocomposites. Piezoelectric $\beta$ Polymorph Formation. Polymer, 51, 5846-5856.

[18] M.A. Rafiee, J. Rafiee, Z. Wang, H. Song, Z.Z. Yu, and N. Koratkar. (2009). Enhanced Mechanical Properties of Nanocomposites at Low Graphene Content. ACS. Nano, 3, 3884-3890.

[19] T. Ramananthan, A.A. Abdala, S. Stankovich, D.A. Dikin, M. Herreea Alonso, R.D. Piner, D.H. Adamson, H.C. Schniepp, X. Chen, R.S. Ruoff, S.T. Nguyen, I.A. Aksay, R.K. Prud'homme, and L.C. Brinson. (2008). Functionalized graphene sheets for polymer nanocomposites. Nature Nanotechnology, 3(6), 327-331.

[20] G. Eda, and M. Chhowalla. (2009). Graphene-based Composite Thin Films for Electronics. Nano Letters, 9(2), 814-818.

[21] H. Kim, and C.W. Macosko. (2009). Processing property relationships of polycarbonate/graphene composites. Polymer, 50(15), 3797-3809.

[22] J. Liang, Y. Huang, L. Zhang, Y. Wang, Y. Ma, T. Guo, , and Y.Chen. (2009). Molecular Level Dispersion of Graphene into Poly (vinyl alcohol) and Effective Reinforcement of their Nanocomposites. Advanced Functional Material, 19(14), 2297-2302.

[23] P. May, A. Khan, O’Neill and J.N. Coleman. (2012). Approaching the theoretical limit for reinforcing polymers with graphene. Journal of Materials Chemistry, 22(4), 1278-1282.

[24] M.A. Rafiee, J. Rafiee, I. Srivastana, Z. Wang, H. Song, Z.Z. Yu, and N. Koratkar. (2010). Fracture and Fatigue in Graphene Nanocomposites. Small, 6(2), 179-183.

[25] J.N. Israelachvili. (2011). Interaction of Biological Membranes and Structures. Intermolecular and Surface Forces, Elservier, 577-616.

[26] L.Y. Jiang, Y. Huanga, H. Jiang, G. Ravichandran, Gao H., K.C. Hwang and B. Liu. (2006). A Cohesive Law for Carbon Nanotube/Polymer interfaces based on the Van Der Waals Force. Journal of the Mechanics and Physics of Solids, 54(11), 2436-2452.

[27] K. Hu, D. Kulkarni, I. Choi, and V. Tsukru. (2014). Graphene-Polymer Nanocomposites for Structural and Functional Applications. 39(11), 1934-1972.

[28] B. Shen, W. Zhai, C. Chen, D. Lu, J. Wang, and W. Zheng. (2006),

Melt Blending In Situ Enhances the Interaction between Polystyrene and Graphene through $\pi-\pi$ Stacking. Journal of the Mechanics and Physics of Solids, 54(11), 2436-2452.

[29] H.L. Zhang, X.L. Wei, Y. Zang, J.Y. Cao, S. Liu, X.P. He, Q. Chn, Y.T. Long, J. Li, G.R. Chen, and K. Chen. (2013). Fluorogenic Probing of Specific Recognitions between Sugar Ligands and Glycoprotein Receptors on Cancer Cells by an Economic Graphene Nanocomposite. Advanced Materials, 25(4097), 101.

[30] P. Costa, J. Nuns Pereira, J. Oliveirs, J. Silva, J. Agostinho Moreira, S.A.C. Carabineiro, J.G. Buijnsters, and S. Lanceros Mendez. (2017). High-Performance Graphene-Based Carbon Nanofiller/Polymer Composites for piezoresistance sensor application. Composite Science and Technology, 153(Nov), 241-252.

[31] J. Liang, Y. Huang, L. Zhang, Y. Wan, , Y. Ma, T. Guo and Y. Chen. (2009). Molecular Level Dispersion of Graphene into Poly (vinyl alcohol) and Effective Reinforcement of their Nanocomposites. Advanced Functional Materials, 19(14), 2297-2302.

[32] V. Sharma, M. Goyal, and P. Jindal. (2017). Characterization and Study of Mechanical Properties of Graphene/ABS Nanocomposites. Indian Journal of Science and Technology, 10, 1-5.

[33] K. Zhang, L.L. Zhang, X.S. Zhao, and J. Wu. (2010) Graphene/Polyaniline Nanofiber composites as supercapacitor electrodes. Chemistry of Materials, 22(4), 1392-1401.

[34] H. Kim, Y. Miura, and C.W. Macosko. (2010). Graphene / Polyurethane Nanocomposites for Improved Gas Barrier and Electrical Conductivity. Chemistry of Materials, 22(11), 3441-3450.

[35] D.A.C. Brownson, D.K. Kampouris and C.E. Banks. (2011). An Overview of Graphene in Energy Production and Storage Applications. Journal of Power Sources, 196(11), 4873-4885.

[36] X. Huang, X. Qi, F. Boeyab and H. Zhang. (2012). Graphene-Based Composites Society Reviews, 41(2), 666-686.

[37] S.V. Morozov, K.S. Nanoselov, M.I. Katsnelson, F. Schedin, D.C Elias, J.A. Jaszczak and A.K. Geim. (2008). Giant Intrinsic Carrier Mobilities in Graphene and Its Bilayer. Physic Review Letter, 100(1), 11-14 [38] Y.M. Lin, C. Dimitrakopoulos, K.A. Henkins, D.B. Farmer, H.Y. Ghiu, A. Grill and P. Avouris. (2010). $100 \mathrm{GHz}$ Transistors from Water Scale Epitaxial Graphene. Nano, 327(5996), 100

[39] X. Li, W. Cai, J. An, S. Kim, J. Nah, D. Yang, R. Piner, A Velamakanni, I. Jung, E. Tutuc, S.K. Banerjee, L. Colombo and R.S. Ruof (2009). Large Area Synthesis of High Quality and Uniform Graphene Films on Coper Foils. Science, 324(2932), 3.

[40] F. Schedin, A.K. Geim, S.V. Morozov, E.W. Hill, P. Blake, M.I. Katsneson and K.S. Novoselov. (2007). Detection of Individual Gas Molecules Adsorbed on Graphene. Nature Materials, 6(9), 652-655.

[41] K. Novoselov and A. Geim. (2007). Graphene Detects Single Molecule of Toxic Gas. Materials Technology, 22, 179-179.

[42] A. Tzalenchuk, S. Lara Avila, A. Kalaboukhov, S. Paolillo, M Syyajarvi, R. Yakimova, O. Kazakoya, T. Janssen, V. Fal'ko and S Kubatkin. (2010). Towards a quantum resistance standard based on epitaxial graphene. Nature Nanptechnology, 5, 186.

[43] L. Lio, J. Bai, Y. Lin, Y. Qu and Y. Huang. (2010). High-Performance Top-Gated Graphene-Nanoribbon Transistors Using Zirconium Oxide Nanowires as High-Dielectric-Constant Gate Dielectrics. Advanced Materials, 22, 1941
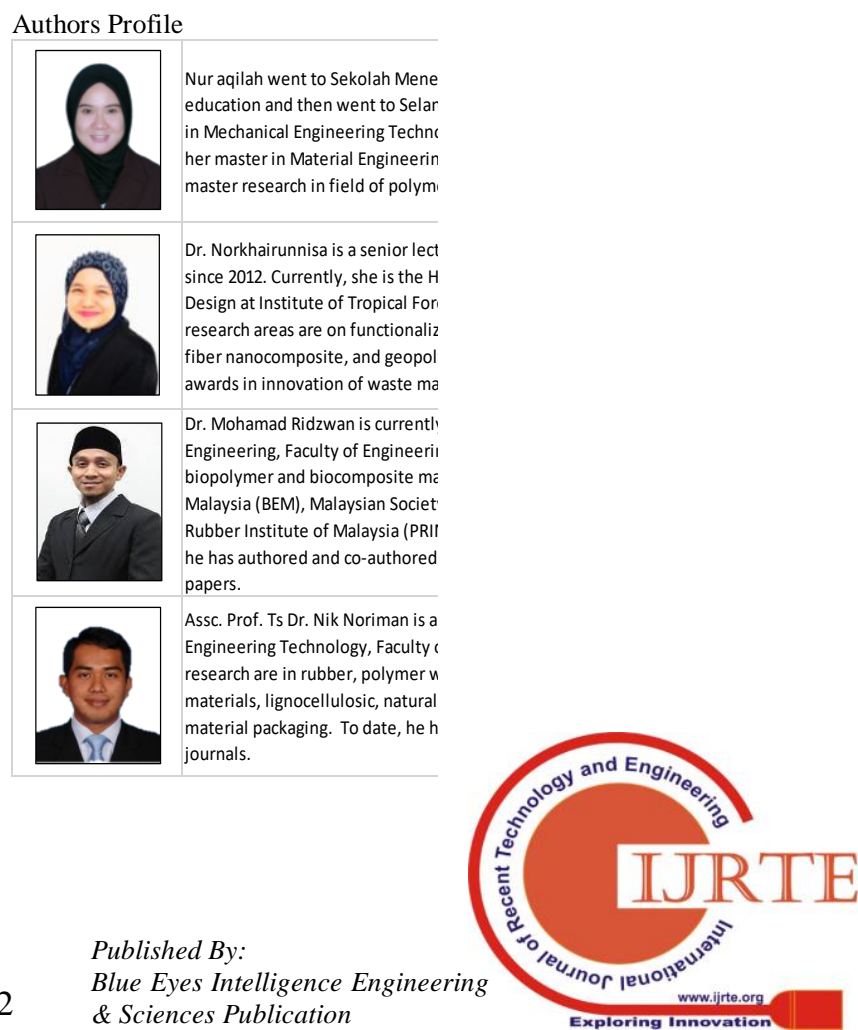